
- FIG 1: In a Quantum Hall insulator, electrons can carry current from contact to contact (a) even though they cannot pass through the bulk, by bouncing along the edge. The states on the edge form discrete channels, which can be revealed by the dispersion relation (b) of a part of the sample with translation invariance along $x$ (dotted rectangle in (a)). Energy eigenstates localized near the upper (red) and lower (blue) edge of the sample form branches of the dispersion relation, and show that propagation along each edge is in one direction only. on each edge, which lead to a very precisely ( 9 significant digits) quantized value of Hall conductance: $U_{\text {transverse }}=$ $n I 2 e^{2} / h$, as revealed by 4 - and 6-terminal measurements.

Having a discrete integer associated to a bulk insulator hints at a topological invariant hiding behind surface state conduction in the Quantum Hall Effect. The explanation was given in the first years of the 1980's, and was part of the reason Thouless was awarded the Nobel prize in physics in 2016. Thouless et al. have shown[7] that the number of edge state channels, i.e., open channels at the boundary of the sample, is given by the net number of topological defects (of the Berry connection) in the Brillouin Zone, counted by the first Chern number of the occupied bulk bands.

In the decades since the discovery of the Quantum Hall Effect more examples have been found where robust edge states appear in tandem with nontrivial bulk Brillouin-zone topology. Haldane (another of 2016's Nobel laureates) has shown using a toy model[8] that a net external magnetic field is not required. A large boost to this line of research was given by Kane and Mele [9] who have shown that these phenomena do not require any breaking of time-reversal symmetry, which was experimentally demonstrated $[10,11]$ on a thin layer of $\mathrm{HgTe}$.

\section{Topological invariants of band insulators}

The reason why some insulators are "topological" is that they have topological invariants. A topological invariant is an integer-valued property of the sample that cannot change under so-called adiabatic deformations: a continuous change of either the sample's shape or its parameters, with the bulk gap not closed, and the relevant symmetries respected. Topological invariants thus represent obstructions against adiabatically deforming the sample to an atomic insulator - a material that consists of disconnected unit cells. The topological invariants come in two flavours: those associated to the boundary of the sample and those associated to the bulk.

The boundary topological invariants of topological insulators quantify the robust physical properties of the surface of the sample. The invariant is essentially the number of protected surface states. For topological insulator wires, it is the number of edge states with protected energy, for two-dimensional topological insulators, the value of surface conductance in units of the conductance quantum, while in three-dimensional case, it is the number of Dirac cones on the surface. The surface states are topologically protected in the sense that their number (or other properties specified by the invariant) cannot change under adiabatic deformations. Topologically protected surface states constitute an obstruction against adiabatic deformation to an atomic insulator, since in atomic insulators the surface is no different from the bulk, and so they cannot host surface states in the bulk gap.

The bulk topological invariants are easiest to define for disorder-free, noninteracting systems, where they can be accessed through the description of the energy bands. Each energy band is a mapping from the toroidal Brillouin Zone to the internal Hilbert space, since each plane-wave energy eigenstate not only has an energy but also some orbital and spin structure. This mapping is smooth, but can have topological defects, which can present obstructions to smoothly connect the mapping to the trivial mapping, i.e., where all plane waves in the band have the same orbital structure, corresponding to the atomic insulator. It is the number of these topological defects, e.g., twists, vortices, skyrmions, of some function of occupied bands over the Brillouin Zone, that is the topological invariant of the bulk - for an example, see Fig. 2. When the bulk is disordered, defining its topological invariants becomes more tricky, and can be done, e.g., using suitably defined scattering matrices[12].

\section{Bulk-boundary correspondence}

The two invariants of topological insulators, that of the surface, and that of the bulk, are connected by the bulk-boundary correspondence, which is at the heart of the theory of topological insulators. For the Quantum Hall Effect, this correspondence is the finding of Thouless that the net number of edge state channels is given by the Chern number of the bulk. This type of connection persists for topological insulators of any dimension or symmetry class, e.g., between the number of topologically protected edge state channels and the bulk $Z_{2}$ invariant in time-reversal invariant two-dimensional

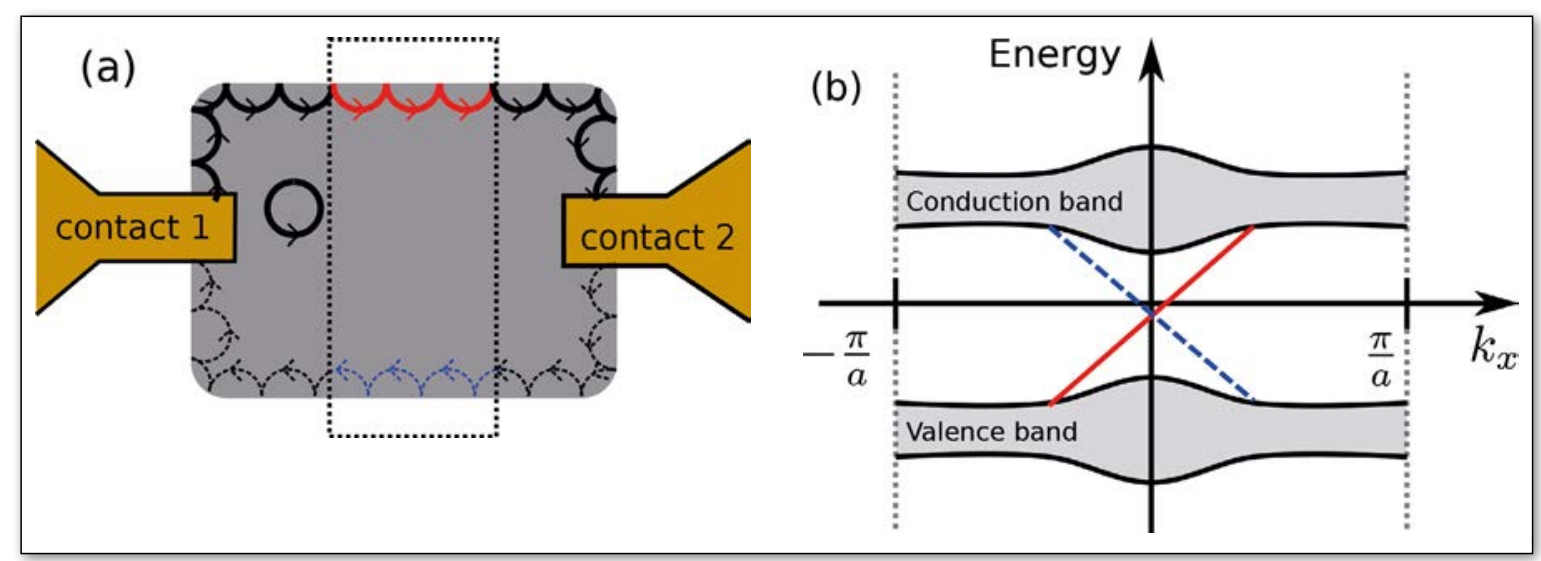




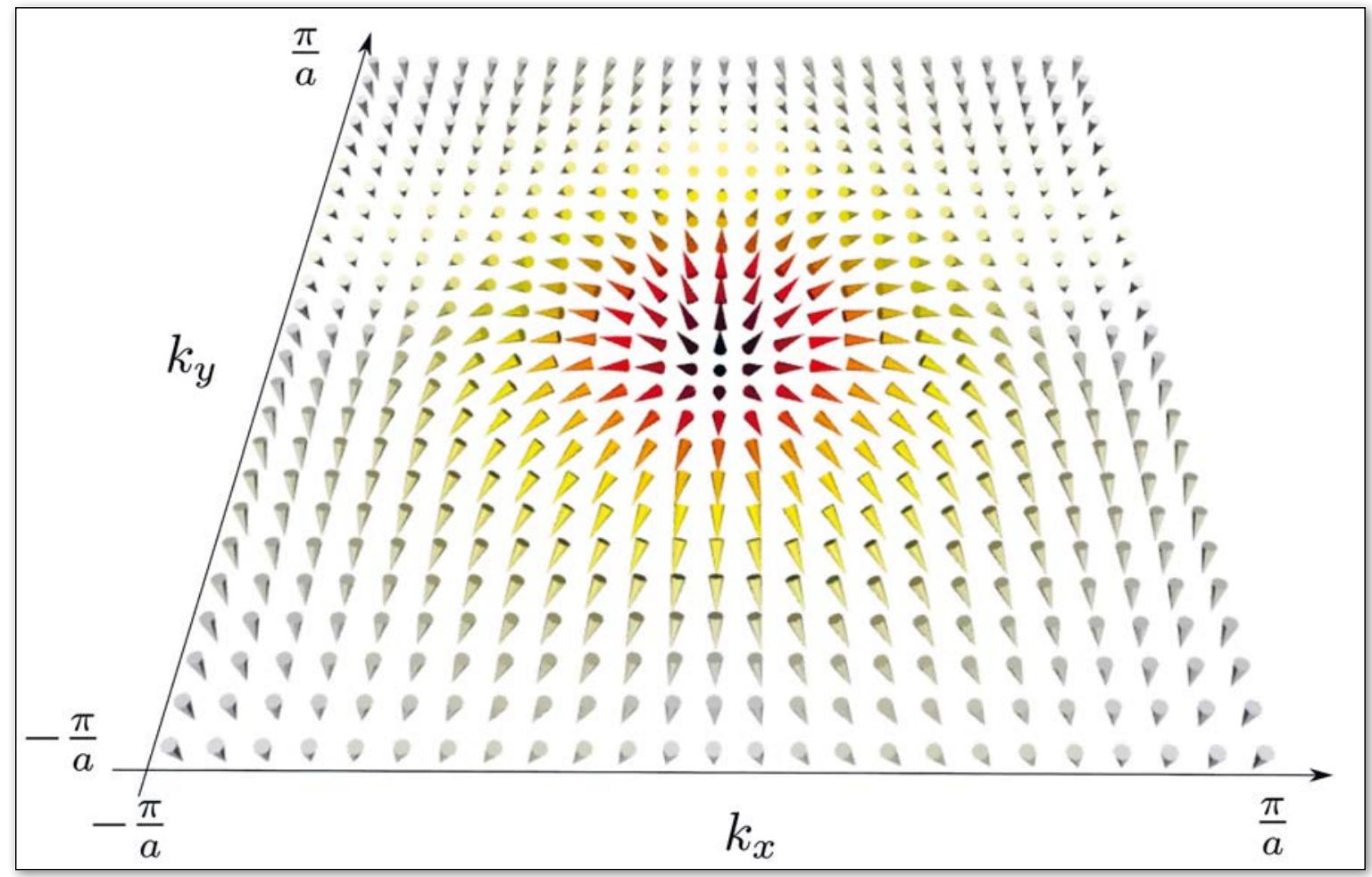

topological insulators, or the bulk sublattice polarization and the number of edge states in the Su-Schrieffer-Heeger model for polyacetylene. Bulk-boundary correspondence is often loosely stated as a topological phase transition in space. Since there is no way to adiabatically deform insulators with different bulk topological invariants into each other, at a smooth interface between them, where the parameters are varied slowly in space, the bulk gap must close and hence there have to be edge states. Nevertheless, turning this loose argument into a precise statement is not straightforward, as bulk-boundary correspondence should apply to the physical edges of the material (boundaries with vacuum) as well as to interfaces where the spatial variation of the parameters is abrupt. Thus, bulk-boundary correspondence becomes a deep statement, requiring more complicated tools, such as connecting charge pumping with edge states[13], and whose rigorous proof is ongoing work.

\section{Topological superconductors, and the full classification}

Superconductors can also display the bulk-boundary correspondence characteristic of topological insulators, if instead of the electric charge, one considers more general single-particle excitations. Although an electron can travel across a superconductor, it can only do so by taking another electron with it with which it can form a Cooper pair. In a mean-field picture this happens via a so-called Andreev reflection, where an incident electron is reflected as a hole. A superconductor is then described in an extended space of electrons and holes as a band insulator, by the so-called Bogoliubov-de Gennes Hamiltonian. Bulk topological invariants can then be associated to superconductors from the energy bands of the Bogoliubov-de Gennes
Hamiltonian (or using the scattering matrices or other approaches). The corresponding topologically protected surface states are electron-hole superpositions, which have a close analogy with Majorana fermions[14]. Such solid-state Majorana fermions can be created and manipulated in semiconducting quantum wires proximitized by ordinary superconductors. Because of the topological protection of the energies of these states, they are seen as a promising path for building a quantum computer[15].

The complete classification of universality classes of topological insulators and superconductors[16-18] was one of the milestones of condensed matter theory in the previous decade. For band insulators of any dimensions it has been identified what combination of nonspatial symmetries (time-reversal, particle-hole, or chiral symmetry) are required the system to be a topological insulator. Derivations of the classification use dimensional extension/ reduction[12,16-18] to connect topological insulators of different dimensionality. Because the requirements follow an 8-dimensional periodicity, the classification is known as the periodic table of topological insulators.

\section{Extensions of the field of topological insulators}

An actively researched extension of the field of topological insulators is topological semimetals[19]. These are zero-gap semiconductors where the conduction and valence bands touch each other at topologically protected, isolated points (or lower dimensional manifolds) of degeneracy in the Brillouin zone. To see that the degeneracies in such materials are protected, one calculates the topological invariant associated to a closed surface in the Brillouin zone that encloses the degeneracy. If the invariant is nonzero, then it
4 FIG 2:

A topological defect, a skyrmion in the Brillouin zone of a two-band toy model for a topological Chern Insulator (Qi-Wu-Zhang model, with $\mathrm{u}=1.5$ using the notation of [13]). The arrows are vectors on the Bloch sphere, representing the orbital structure of the occupied band. Bearing in mind the periodic boundary conditions, there is no smooth deformation of this field into a trivial one, corresponding to an atomic insulator, where all arrows face in the same direction.(Source: László Oroszlány). 
represents an obstruction to a local adiabatic deformation of the system that would separate the two bands. Around the degeneracy points the dispersion relation is linear, and such materials host analogues of Weyl fermions in the same way as topological superconductors do for Majorana fermions.

Another direction in which the field of topological insulators is pushed is to incorporate spatial symmetries, such as inversion, or rotation. So-called crystalline insulators are materials whose topological invariants depend crucially on such symmetries. They host topologically protected surface states only on surfaces that are aligned with the crystallographic axes so that the important spatial symmetries are respected. Incorporating the various types of symmetries to produce greater and greater periodic tables of topological crystalline insulators is ongoing work[3].

Floquet topological insulators[20] are periodically driven systems that can host edge states even if all of their bulk bands have trivial topology. Periodic driving, or the "shaking of the lattice" on timescale much shorter than the timescale of the dynamics is used in experiments on ultracold atoms trapped in optical lattices to imprint complex phases on the hopping amplitudes, and thus simulate topological insulators[21]. The effects of a drive with a timescale longer than that of the dynamics is more difficult to describe, and it is in this limit that topologically protected edge states can arise even if the topological invariants of the quasienergy bands of the effective Hamiltonian are all trivial[22]. The simplest toy models that display such novel topological phases are quantum walks[23,24].

Although the theory of topological insulators relies on quantum physics, many of the concepts carry over to classical mechanics. Specially engineered systems of springs and sticks can contain topologically protected modes on the edges[25] or at defects[26], and concepts of topological insulators have been invoked to explain the origin of equatorial waves in the ocean and the atmosphere[27].

In the last thirty-odd years, topological insulators have had a lasting impact on condensed matter physics. They have offered us a glimpse into the rich world of phase transitions that are not driven by a change in symmetry, but rather in topology. Moreover, the defining physical processes in topological insulators are "superficial", they take place on the boundary of a material. Nevertheless, they are connected to the topological nature of the depths of the material, of the bulk. Thus, in topological insulators, the deep controls the superficial.

\section{About the Author}

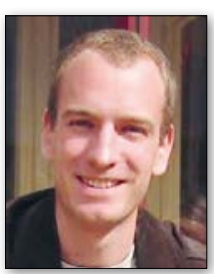

János K. Asbóth is a senior research fellow at the Department of Quantum Optics and Quantum Information of the Wigner Research Centre for Physics of the Hungarian Academy of Sciences, Budapest. His research has focused on topological phases of quantum walks. He has been giving university courses on topological insulators in Budapest, Geneva, and Bonn, and co-written a Springer Lecture Note in Physics on the topic (A Short Course on Topological Insulators, with András Pályi and László Oroszlány, 2016).

\section{References}

[1] M. Z. Hasan and C. L. Kane, Reviews of Modern Physics 82, 3045 (2010).

[2] X.-L. Qi and S.-C. Zhang, Reviews of Modern Physics 83, 1057 (2011).

[3] C.-K. Chiu, J. C. Teo, A. P. Schnyder, and S. Ryu, Reviews of Modern Physics 88, 035005 (2016).

[4] Y. Ando, Journal of the Physical Society of Japan $\mathbf{8 2 ,}$ 102001 (2013).

[5] W. Su, J. Schrieffer, and A. J. Heeger, Physical Review Letters 42 1698 (1979).

[6] S.-C. Zhang and J. Hu, Science 294, 823 (2001).

[7] D. J. Thouless, M. Kohmoto, M. P. Nightingale, and M. Den Nijs, Physical Review Letters 49, 405 (1982).

[8] F. D. M. Haldane, Physical Review Letters 61, 2015 (1988).

[9] C. L. Kane and E. J. Mele, Physical Review Letters 95, 146802 (2005).

[10] B. A. Bernevig, T. L. Hughes, and S.-C. Zhang, Science 314 1757 (2006).

[11] M. König, S. Wiedmann, C. Brüne, A. Roth, H. Buhmann, L. W. Molenkamp, X.-L. Qi, and S.-C. Zhang, Science 318, 766 (2007).

[12] I. C. Fulga, F. Hassler, and A. R. Akhmerov, Physical Review B 85, 165409 (2012)

[13] J. K. Asbóth, L. Oroszlány, and A. Pályi, Lecture Notes in Physics 919 (Springer, 2016).

[14] C. Beenakker, Annual Review of Condensed Matter Physics 4, $113(2013)$

[15] J. Alicea, Y. Oreg, G. Refael, F. Von Oppen, and M. P. Fisher, Nature Physics 7, 412 (2011).

[16] A. Kitaev, in AIP Conference Proceedings (AIP, 2009) 1134, pp. 22-30.

[17] S. Ryu, A. P. Schnyder, A. Furusaki, and A. W. Ludwig, New Journal of Physics 12, 065010 (2010).

[18] J. C. Teo and C. L. Kane, Physical Review B 82, 115120 (2010).

[19] N. Armitage, E. Mele, and A. Vishwanath, arXiv preprint arXiv:1705.01111 (2017).

[20] J. Cayssol, B. Dóra, F. Simon, and R. Moessner, physica status solidi (RRL) 7, 101 (2013).

[21] N. Goldman, G. Juzeliūnas, P. Öhberg, and I. B. Spielman, Reports on Progress in Physics 77, 126401 (2014).

[22] M. S. Rudner, N. H. Lindner, E. Berg, and M. Levin, Physical Review X3, 031005 (2013).

[23] J. K. Asboth and J. M. Edge, Physical Review A 91, 022324 (2015).

[24] T. Groh, S. Brakhane, W. Alt, D. Meschede, J. Asbóth, A. Alberti, Physical Review A 94, 013620 (2016)

[25] C. Kane and T. Lubensky, Nature Physics 10, 39 (2014).

[26] J. Paulose, B. G.-g. Chen, and V.Vitelli, Nature Physics 11, 153 (2015).

[27] P. Delplace, J. Marston, and A. Venaille, Science 358, 1075 (2017). 